\title{
GW23-e1073 C-MET OVEREXPRESSION PROMOTE REENDOTHELIALISATION AND INHIBIT NEOINTIMAL FORMATION AFTER BALLOON INJURY
}

doi:10.1136/heartjnl-2012-302920a.36

Song Mingbao, Shi Yankun, Zhao Gang, Yu Xuejun, Chen Jianfei, Huang Lan. Institute of Cardiovascular Diseases of PLA, Xinqiao Hospital, Third Military Medical University

Objectives to explore the effect of c-met overexpression in EPCs on reendothelialisation after balloon injury

Methods EPCs derived from mouse bone marrow were isolated and cultured. 3-(4,5-dimethylthiazol-2-yl)-2,5-diphenyltetrazolium bromide assays were used to evaluate EPC proliferation. Adenoviral vector expressing c-Met was generated using the AdEasy system. To evaluate the role of HGF/Met in vascular repair in vivo, we used balloon-injured rat carotid artery model. Evans Blue dye was administered to evaluate reendothelialisation after 10 days injury, and the neointimal formation was assessed at 21 days following vascular injury.

Results The effect of HGF on EPC proliferation was examined $48 \mathrm{~h}$ after exposure to different quantities of HGF (range 2-20 ng/ml). The proliferation effect was strongly dose-dependent and significantly increased in c-met-EPCs group compared with EPCs group. After transfusion of c-met-EPCs or EPCs to balloon-injured rat via vessel, Evans Blue dye was administered to evaluate reendothelialisation after balloon injury. reendothelialised area was significantly larger in c-metEPCs group than in EPCs group $(64.25 \pm 8.90 \%$ vs $43.21 \pm 7.24 \%, n=5$, $\mathrm{p}<0.01)$. A marked decrease in the neointimal area and $\mathrm{I} / \mathrm{M}$ ratio was found in c-met-EPCs compared with EPCs group at day 21(0.29 \pm 0.06 vs $0.63 \pm 0.13, n=5, p<0.01)$.

Conclusions c-Met overexpression improve EPCs proliferation, promote reendothelialisation and inhibit neointimal formation after balloon injury. 